\title{
Search for significant background variations in the EUSO-Balloon data
}

\author{
A. Jung ${ }^{* 1}$, E. Parizot ${ }^{1}$, G. Suino ${ }^{2}$, M. Bertaina ${ }^{2}$, P. Gorodetzky ${ }^{1}$, P. Barrillon ${ }^{3}$ \\ for the JEM-EUSO collaboration \\ ${ }^{1}$ Laboratoire AstroParticule et Cosmologie - Université Paris Diderot-Paris 7, CNRS/IN2P3 \\ ${ }^{2}$ Dipartimento di Fisica, Università di Torino, \& INFN Torino \\ ${ }^{3}$ Laboratoire de l'Accélérateur Linéaire, Université Paris-Sud, CNRS/IN2P3
}

The EUSO-Balloon instrument recorded data during a stratospheric flight in August 2014, to measure the UV background during a moonless night, from an altitude of $\sim 38 \mathrm{~km}$, with a field of view of $\pm 5.5^{\circ}$. In this paper, we report on the search for coherent fluctuations of this background over areas ranging from $\sim 1 \mathrm{~km}^{2}$ up to the entire field of view, on timescales from a few $\mu$ s to $\sim 100 \mu \mathrm{s}$. In addition to the expected laser track events induced by shootings from a helicopter flying in the field-of-view of EUSO-Balloon during part of the mission, three unidentified and significant events, probably related to human activity, are discussed.

The 34th International Cosmic Ray Conference,

30 July- 6 August, 2015

The Hague, The Netherlands

${ }^{*}$ Speaker. 


\section{Introduction}

The EUSO-Balloon [1] instrument has been conceived as a prototype to the JEM-EUSO space mission $[2,3]$. Both are dedicated to the study of ultra-high-energy cosmic rays (UHECRs) from space or near space via detection of the fluorescence emission caused by the extensive air shower generated when an UHECR collides with the atmosphere. The EUSO-Balloon instrument consists of a UV telescope with Fresnel optics giving a field of view of $\pm 5.5^{\circ}$, and a focal surface equipped with 36 multi-anode photomultiplier tubes (MAPMT) of 64 pixels each, with single photon sensitivity. The instrument has been designed to fly under a stratospheric balloon at $\sim 38 \mathrm{~km}$ altitude. The mission was funded by the French National Center for Space Studies, CNES, with contributions of the international JEM-EUSO collaboration for various subsystems (see $[4,5]$ for details about the mission, the flight and the instrument performance).

On the night of the 24th-25th August, EUSO-Balloon took data in the 290-430 nm UV band over a varying landscape and with variable cloud coverages using a variety of different data taking modes. One goal of the mission was to demonstrate the capability of the instrument to measure the UV background from space under various conditions to better estimate the performance of the planned JEM-EUSO space-based UHECR telescope (see [6]). Given the short data taking time and EUSO-Balloon's energy threshold for cosmic-ray shower detection around $10^{18} \mathrm{eV}$, no actual cosmic-ray detection was expected. To remedy the absence of such interesting physical events, a helicopter was flown under the balloon for a period of $\sim 2$ hours, flashing intense light sources and shooting horizontal laser beams whose Rayleigh-scattered light was susceptible to be detected by the EUSO-Balloon telescope (see [7] for details).

The standard analysis procedure for cosmic-ray shower detection consists in looking for signals with a consistent space-time structure over the focal surface, corresponding to the development of showers with an arbitrary, but definite direction with respect to the instrument's optical axis. A specific trigger has been designed and optimized to detect and select such signals in the focal surface of JEM-EUSO and EUSO-Balloon. Its application to the EUSO-Balloon data is presented in a separate paper [8].

Apart from signals associated with cosmic rays (CRs), other potential signals include meteors and lightning as well as artificial lights. Most of these signals are not expected to show strong variations over sub-millisecond timescales. Various types of so-called transient luminous events (TLEs) are also known to originate from the ionosphere (UV flashes, elves, sprites, blue jets, etc. [9]). Although they are not expected to be observed directly by EUSO-balloon, since they are produced at higher altitudes, some of the signals emitted in the ionosphere might in principle be observed as reflected light, especially above clouds, which have a higher UV albedo.

The data collected during the flight are the first (known) measurements of the Earth's UV emission (or reflection) with a focusing instrument with small scale resolution. Since very few studies have been devoted to this UV signal, it is interesting, as a first step, to search for very generic, unspecified events which may manifest themselves as variations of the UV light flux observed in the nadir direction, over tens or hundreds of $\mu \mathrm{s}$, as allowed by the time resolution of EUSO-Balloon. In this paper, we present the preliminary results of such a study. 

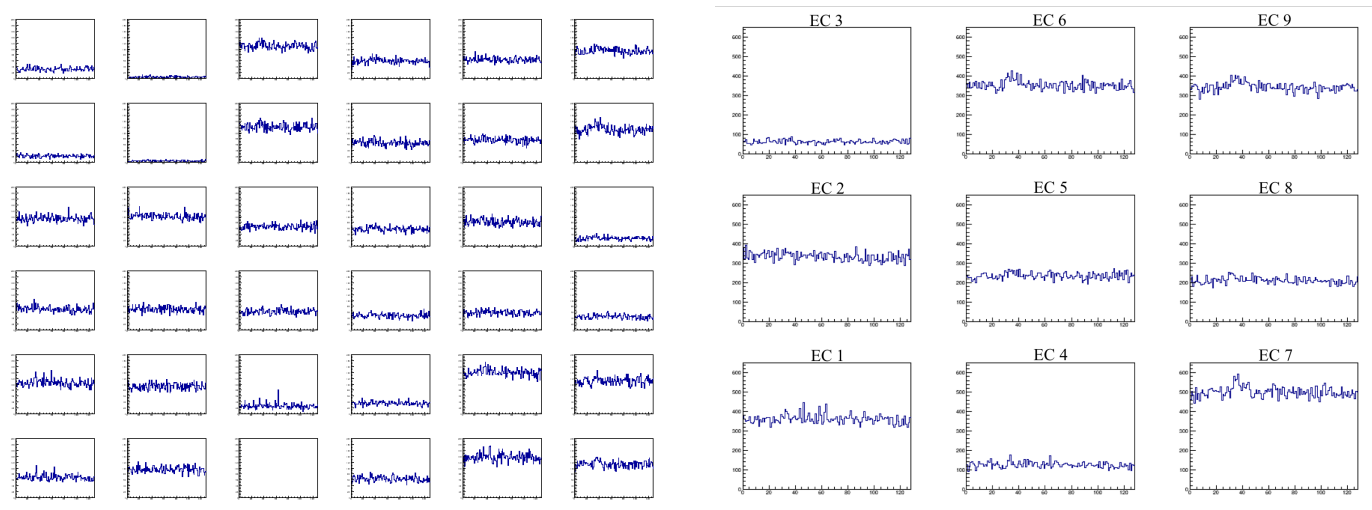

Figure 1: Total photon count in each of the MAPMTs (left) and each of the ECs (right) of the focal surface of EUSO-Balloon, as a function of time, for one data packet. The $\mathrm{x}$-axis is in GTUs (2.5 $\mu$ s unit). The y-axis range is from 0 to 200 for PMT signals, and from 0 to 650 for EC signals.
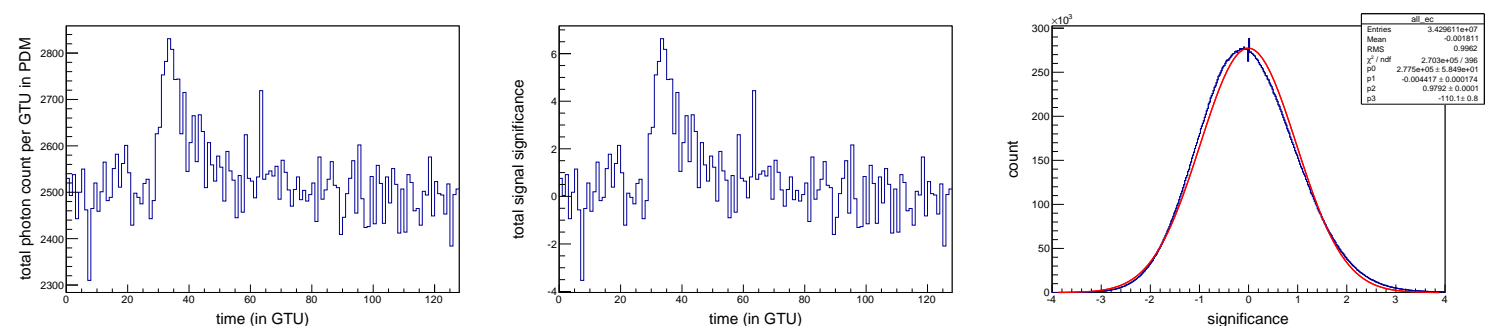

Figure 2: Total photon count of the entire PDM (left) and corresponding signal significance (center), as a function of time (in GTUs). Right: Histogram of the significance of the photon count in the PDM, for all the periods considered without signal (see text), together with a Gaussian fit.

\section{The method}

The EUSO-Balloon data collected during the flight are organized in a number of separate files, containing so-called packets of data, each of which contains the photon count registered for each of the 2304 pixels in the focal surface during 128 consecutive time bins. Each time bin is called a gate time unit (GTU). GTUs have a constant duration of $2.5 \mu \mathrm{s}$. Therefore, 1 packet corresponds to a period of $0.32 \mathrm{~ms}$. The focal surface will be referred to as the photo-detection module (PDM). It is subdivided into 9 so-called elementary cells (ECs), consisting of $2 \times 2$ matrices of MAPMTs.

Figure 1 shows an example of the photon count per GTU for one packet, obtained by summing the photon counts of all pixels in each of the 36 MAPMTs (left) and in each of the 9 ECs (right). As can be seen, the total count rate is quite different on average for different ECs. This is because of some defective PMTs and electronics failures (as can be also seen on the left panel).

In the present study, we are interested in significant variations of the photon count over the whole PDM (entire focal surface) or individual ECs. The left panel of Fig. 2 shows the evolution of the PDM signal during one data packet (128 GTUs). A signal can be clearly seen around GTU number 35 . To perform a systematic study of such signals, we proceed as follows. 
First, we divide the time interval into 3 parts, containing respectively 43, 43 and 42 GTUs. We determine the average photon count per GTU during each of these periods, as well as its RMS, $\sigma$. We keep the smallest average value as indicative of the average UV background during the whole period, and the corresponding RMS is used to determine the significance of the photon count fluctuations. We then produce a normalized photon count evolution by plotting the so-called significance signal, defined for GTU number $i$ in packet number $p$, as:

$$
\Sigma_{i}=\frac{S_{i}-A_{p}}{\sigma_{p}}
$$

where $S_{i}$ is the original signal (photon count) in the PDM at GTU number $i$, and $A_{p}$ and $\sigma_{p}$ are the average and RMS adopted for the packet under consideration.

The center panel of Fig. 2 shows the significance signal for the same period as in the left panel. As expected by construction, the new average signal is 0 , and the new RMS is 1 . In this way, we can treat in a consistent way all the signals in all the data packets, and we can compare the signals recorded by different ECs, even though they do not have the same photon detection efficiencies (see above).

In the next step, we search the whole data set in a systematic way. We look for significant upward fluctuations of the signal by requiring at least $N=3$ consecutive GTUs with a photon count more than $T$ sigmas away from the average, i.e. with a signal significance $\Sigma_{i} \geq T$. We call an "event" a sequence of signals satisfying this criterion. In the following, we use $T=3$ as the default value for the significance threshold, but we also explored other combinations of $N$ and $T$ values to search for potentially fainter, but still significant signals. With the choice $N=T=3$, we can estimate the probability of a "fake event" (i.e. obtained from a random fluctuation of the background). Assuming the total photon count over the entire PDM follows a Gaussian distribution (apart from when "events" are recorded), the probability for the significance signal, $\Sigma$, in one GTU to be larger than 3 is $\mathscr{P}(\Sigma \geq 3) \simeq 1.35 \times 10^{-3} \equiv \mathscr{P}_{0}$. Therefore, the probability of a fake event (beginning at any given time) is $\mathscr{P}_{0}^{3} \simeq 2.5 \times 10^{-9}$. The number of packets recorded during the EUSO-Balloon flight (with nominal configuration) is 123,800. With 128 GTUs each, this amounts to a total of $\sim 1.6 \times 10^{7}$ GTUs. The probability of finding a fake event in the whole data set is smaller than $4 \%$. The right panel of Fig. 2 shows the histogram of the significance values, $\Sigma$, of the total EC photon count per GTU. In this histogram, all the ECs are considered separately, and the data are restricted to the periods which served as a reference for the calculation of the average and RMS in the above-described procedure (1/3 or the total data). As a first approximation, one may consider that these periods are free from significant signals (over the considered timescale), and only show statistical fluctuations of the background count. As can be seen, the distribution is very close to Gaussian (with average 0 and RMS 1, as expected by construction), which supports the assumption that the signal detected in these periods is essentially random background light, and thus gives weight to the above estimate of the fake event rate.

\section{Results}

In total, around 400 events were detected. Most of these events have also been identified by the trigger algorithm specifically designed to detect cosmic-ray tracks in the focal surface. They 

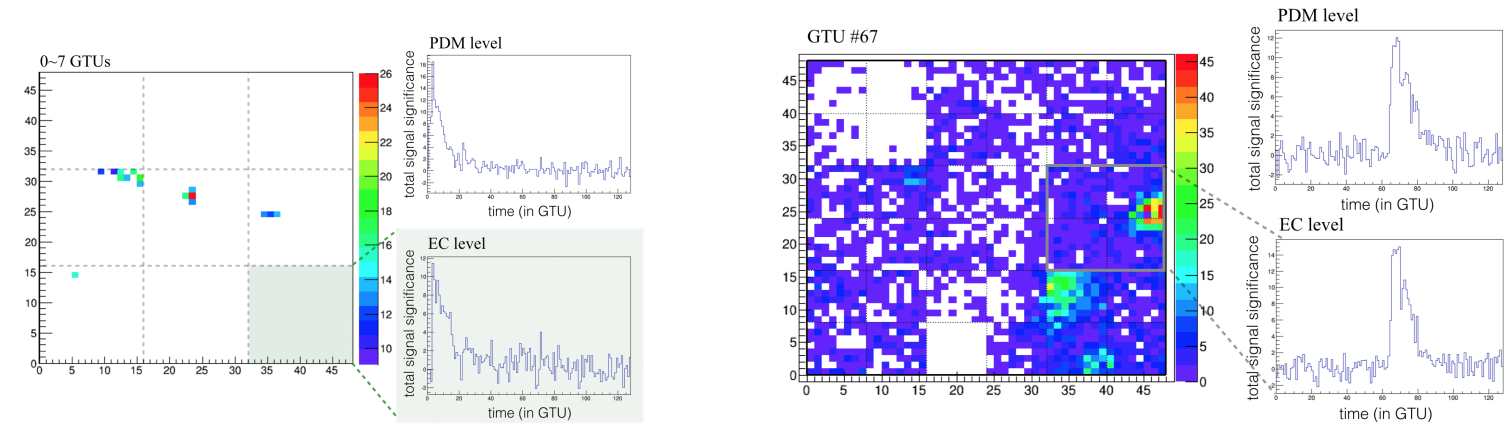

Figure 3: Left: composite image of the photon count in each pixel in the PDM, summed over the first 7 GTUs of the data packet. The corresponding significance signal for the whole PDM and for EC number 7 are also shown. Right: photon count per pixel over the entire PDM (color map) during the recording of a highly significant signal in EC number 8 (bottom right). The significance signal of the whole PDM is also shown (upper right).

correspond to laser tracks crossing the field of view of EUSO-Balloon's telescope [7, 10], and confirm both the ability of the instrument to detect cosmic-ray-like events, and the effectiveness of the algorithm. These events, their detection and their analysis are discussed in separate papers $[10,8]$.

In addition to these well-identified (laser) events, whose print can be followed on the focal surface over several GTUs, a few additional events could be observed in the data. We describe them briefly below.

The first and largest set of such additional events have been recorded during a relatively short period of time, while the helicopter was flying under the balloon. Even though these events were not recognized as actual tracks by the standard track trigger algorithm, there are good reasons to believe they are nevertheless related to the laser shots. It seems indeed likely that the laser shots simply propagated through an optically thick region, leading to a global increase of the photon count in the corresponding area, without definite source location. Several arguments go in favour of this interpretation.

First, the time period of these events corresponds to a period when the infrared camera indicates the presence of clouds in the field of view $[11,12]$. Second, the largest signal significance, responsible for the qualification of the photon count sequence as an "event", appears to be always located in the same region of the focal surface, namely in ECs number 7, 8 and 9. This part of the field of view is the same as where better-defined laser tracks could be spotted about 1 minute after these events, i.e. when the helicopter was still roughly at the same position and shooting in a similar direction (the global pendular movement of the balloon, around and across the vertical axis, was also not very significant at the considered scale at this moment of the flight). Finally, the timescale of the signal appears compatible with the crossing time of the EC at the speed of light, with some margin associated with the azimuth with respect to the square EC and a small tilt angle of the laser direction with respect to the horizontal.

Apart from these events, probably related to laser shots, and a small number of fake events associated with a visible electronics failure, we have identified two interesting signals, plus another 
class of signals probably related to laser tracks. The latter is illustrated by the event shown on the left panel of Fig. 3. A clearly visible laser track can be seen moving from left to right at the speed of light in the raw data (with some missing elements due to dysfunctional pixels). The total significance signal over the entire PDM is plotted as a function of time (in GTUs) in the upper-right panel. It shows very high significances. However, almost at the same time, a highly significant signal has also been recorded on the bottom-right EC (number 7), without clear spatial pattern. Its time structure is also shown. It is tempting to relate this unidentified signal to the operation of the laser, because laser events were detected over a negligible fraction of the total recorded data $\left(<10^{-3}\right)$, so a mere coincidence seems particularly unlikely. However, the region observed by EC number 7 is far from the region where the laser track is observed, and no signal can go from one place to the other within less than a GTU (it takes several GTUs to the laser itself, at the speed of light!). Therefore, if the coincidence in time is not random, it is likely that the photons responsible for the "event" in EC 7 are not originating from the corresponding region in the FoV, but represent some stray light of the laser track photons diffused/reflected inside the instrument itself, and ending up in EC 7. The same pattern (a clear laser track accompanied by some significant, but diffuse signal in a different place of the focal surface) could also be observed in other occasions. This gives weight to the interpretation that such "events" are caused by some "defect" in the optical system, whose point spread function may be more complicated than expected. This will be investigated further in the coming months.

Another interesting event is shown in the right panel of Fig. 3. Its time structure shows a very highly significant signal in EC number 8, for a total duration of $\sim 15$ GTUs, i.e. less than $40 \mu$ s. The place where this event occurs is clearly associated with a mine, whose light can be followed in the field of view of the instrument over a long period of time. It is thus very likely that this signal is somehow related to human activity. However, we could not identify easily a potential source, given the extremely short timescale. In the case of a reflected signal brought serendipitously into the direction of the balloon by the rotation of a reflecting surface, the angular rotation speed would have to be of the order of 16 turns per second to be compatible with a $40 \mu \mathrm{s}$ signal. A more likely cause could perhaps be a very brief discharge in an electrical device on the mine site.

Another similar event has also been recorded prior to the operation of the helicopter, so that it cannot be related to laser shots or light flashes. It is shown in Fig. 4. Although particularly significant and very well located in space and time, this event may unfortunately also be related to human activity, since it is located in an area where mines could be expected to be observed, given the data recorded in previous and subsequent packets. It remains however mysterious why such a very clear and intense signal was detected during $40 \mu \mathrm{s}$, while the involved PMT recorded no signal in the previous and following minute. Again, a brief discharge may be responsible.

\section{Summary and discussion}

We have presented here a first attempt to systematically search for unspecified signals appearing as significant upward fluctuations in the UV light observed from an altitude of $\sim 38 \mathrm{~km}$, with a total field of view of $\sim 50 \mathrm{~km}^{2}$, and over timescales ranging from a few $\mu \mathrm{s}$ to $\sim 100 \mu \mathrm{s}$.

Although the flight of EUSO-Balloon spanned over several hours, the in-flight tests and data taking strategy had been conceived in such a way that only $\sim 2$ minutes of actual data were recorded 

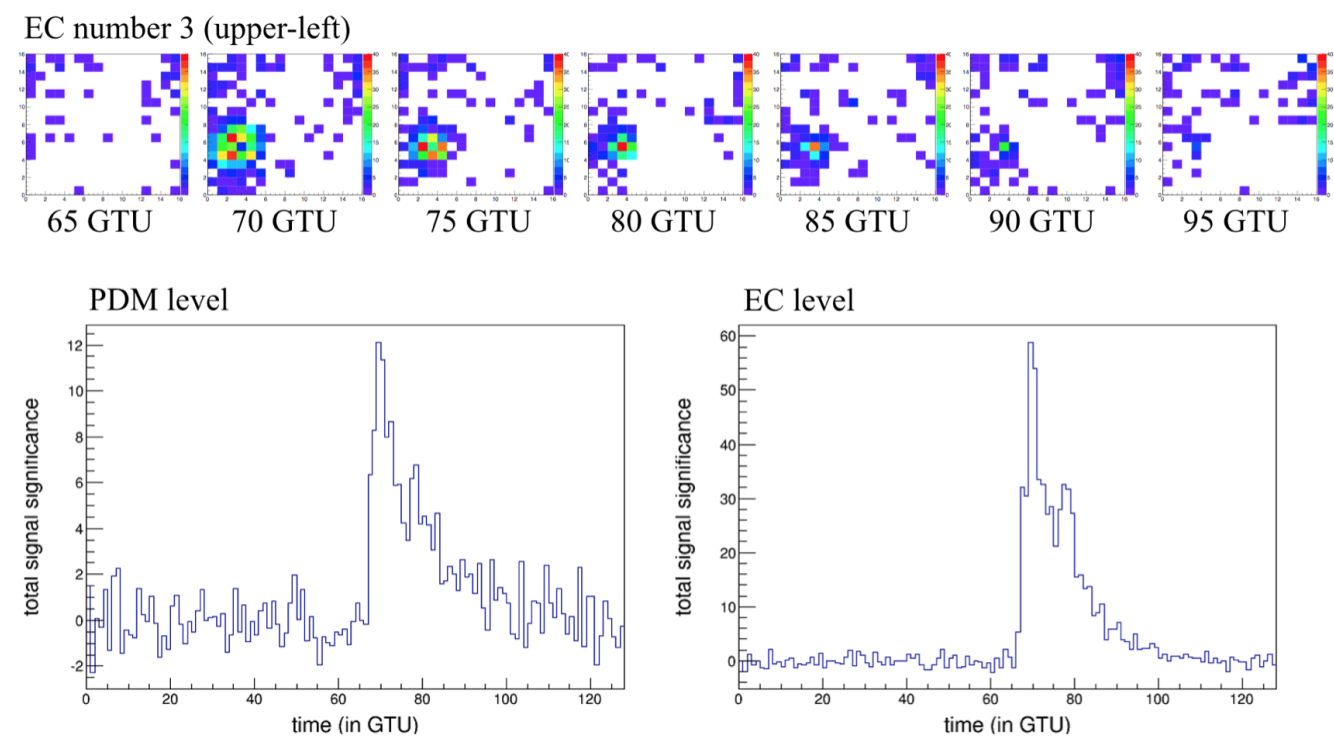

Figure 4: Time sequence (top panels) illustrating the development of a very significant event appearing in EC number 3 (bottom right), over $\sim 40 \mu$ s, clearly visible also at the PDM scale (bottom left), although all the signal comes from a few pixels on one single PMT.

(which was more than enough to measure the UV background and accomplish the objectives of the mission). In such a short observational time, no cosmic-ray showers were expected to be detected, and only relatively frequent events could have a chance to be detected serendipitously.

Apart from the expected laser track events, the search did not lead to the detection of well identified signals. In particular, no meteorological or astronomical events can be reported. Some significant signals nevertheless draw our attention. Two of them are probably associated with human activity, since they are coincident with the position of a mine in the field of view of the instrument. However, their timescale, $\leq 50 \mu \mathrm{s}$, is very short, and their origin remains mysterious. One may be tempted to compare the time sequence of these events, shown in Figs. 3 (right) and 4. Their light curves both show a very sharp increase, which may saturate the dominant pixels, and a roughly exponential decay with a second (weaker peak) after $\sim 10$ GTUs (i.e. $\sim 25 \mu$ s). However, no reliable conclusion can be based on the perhaps fortuitous similarity of only two events, and we simply leave these events as a curiosity here. Further investigations of the instrument response to strong signals will be needed to determine whether a sharp flash of very intense light (perhaps related to some sort of abrupt discharge, like that involved in capacitor discharge ignition systems, with timescales around 50-80 $\mu$ s) could be responsible for such signals.

Another class of events reported here is of a different nature. It appears to be more diffuse in space and much weaker in intensity, although it is clearly significant at the level of one EC. Since it occurred while the helicopter was flying, and was recorded at the same time as a laser shot, which is very unlikely to be purely coincidental, it is tempting to relate these events to the laser. However, the space-time separation between the reconstructed sources of the photons in both (almost) simultaneous events is not compatible with a causal relation between them. One may thus tentatively conclude that the unidentified event accompanying the laser track is due to some stray 
light in the instrument itself, and that the corresponding photons do not come from the region in the atmosphere that is observed by the EC which recorded them, but instead arrived on the pupil of the telescope together with the laser track photons, and were actually part of them.

Finally, we conclude that no significant variation of the UV background over the whole fieldof-view on timescales between 10 and 100 GTUs could be detected.

The next flight of EUSO-Balloon will use a super-pressure balloon (SPB) and will allow continuous data taking over several nights. This should provide data across various conditions of illumination (notably from different moon phases) and cloud coverage, as well as above different types of grounds (mostly over the ocean). This will allow many more studies, and the examination of possible interesting events with a larger statistics, to disentangle between signals of human origin and putative meteorological or astronomical events.

The above results may serve as first guides to design a specific trigger to search for short timescale variations of the background. Based on the present study, it appears that even a simple time-over-threshold trigger procedure at the level of the entire PDM seems to be able to detect events entirely contained in one EC. Longer timescales are also important in principle (astronomical events as well as meteorites and ionospheric events), even though they were not accessible to the present analysis, due to the adopted data taking strategy.

\section{References}

[1] P. von Ballmoos, General overview of EUSO-Balloon mission, in proceedings of the 34th ICRC, POS ( ICRC2015) 0725 .

[2] Y. Takahashi, The JEM-EUSO mission, New J. Phys. 2009, 11, 065009

[3] Fumiyoshi Kajino, The JEM-EUSO mission to explore the extreme Universe, Nuclear Instruments and Methods in Physics Research Section A, 2010, 623 422-424.

[4] A. Jung et al., Design and Implementation of the Photo-Detector Module Electronics for the EUSO-Balloon, Prototype of the JEM-EUSO Telescope, JINST in prep.

[5] G. Osteria, The Data Processor System of EUSO Balloon: in flight performance, in proceedings of the 34th ICRC, POS ( ICRC2015) 1025.

[6] M. Bertaina et al., Performance and air-shower reconstruction techniques for the JEM-EUSO mission, Advances in Space Research 2014, 53 1515-1535

[7] J. Eser, Laser direction reconstruction in EUSOBalloon experiment, in proceedings of the 34th ICRC, POS ( ICRC2015) 0860.

[8] G. Suino, Tests of JEM-EUSO 1st level trigger using EUSO-Balloon data, in proceedings of the 34th ICRC, POS ( ICRC2015) 0925.

[9] G. K. Garipov et al., Global transients in ultraviolet and red-infrared ranges from data of Universitetsky-Tatiana-2 satellite, Advances in Space Research 2013, 118 370-379

[10] M. Bertaina, Preliminary results from the EUSO-Balloon fight, in proceedings of the 34th ICRC, POS ( ICRC2015) 0890.

[11] M. Frias, The Spanish Infrared Camera onboard the EUSO-BALLOON (CNES) flight on August 24, 2015 , in proceedings of the 34th ICRC, POS ( ICRC2015) 1309.

[12] J. Fernandez, Cloud Optical Depth obtained from the Infrared Camera data and the UV Flashers mounted on a helicopter flying under the EUSO-Balloon (CNES) during its flight, in proceedings of the 34th ICRC, POS ( ICRC2015) 1024. 